\title{
Testing the exchange rate data for the parameter change based on ARMA-GARCH model ${ }^{\dagger}$
}

\author{
Junmo Song ${ }^{1} \cdot$ Bangwon $\mathrm{Ko}^{2}$ \\ ${ }^{1}$ Department of Computer Science and Statistics, Jeju National University \\ ${ }^{2}$ Department of Statistics and Actuarial Science, Soongsil University \\ Received 8 August 2013, revised 5 November 2013, accepted 14 November 2013
}

\begin{abstract}
In this paper, we analyze the Korean Won/Japanese 100 Yen exchange rate data based on the ARMA-GARCH model, and perform the test for detecting the parameter changes. As a test statistics, we employ the cumulative sum (CUSUM) test for ARMA-GARCH model, which is introduced by Lee and Song (2008). Our empirical analysis indicates that the KRW/JPY exchange rate series experienced several parameter changes during the period from January 2000 to December 2012, which leads to a fitting of AR-IGARCH model to the whole series.
\end{abstract}

Keywords: ARMA-GARCH model, CUSUM test, exchange rate data, IGARCH model, parameter change.

\section{Introduction}

In statistical modeling, the task of testing for parameter consistency is crucial because ignoring the parameter change can lead to an invalid conclusion. For instance, the example of Hamilton (1994, pp.450) shows that a parameter change can misidentify a stationary AR(1) model for a unstable AR(1) model. Actually, it is well known that economic and financial time series often suffer from structural changes in the underlying models due to occasional changes of monetary policy and critical social events. For these reasons, the change point problem has attracted much attention from many researchers in time series analysis. For relevant reference, we can refer to Wichern et al. (1976), Picard (1985), Kokoszka and Leipus (2000), and Lee et al. (2003). For historical background and general review, see Csörgő and Horváth (1997) and Chan and Gupta (2000), and the articles cited therein.

The class of autoregressive moving average (ARMA) models is very useful for describing the dynamics of individual time series. In financial time series, this class is a natural candidate for conditioning on the past return series. Traditionally, the innovations of ARMA models are assumed to be i.i.d. random variables with mean zero. However, common time

$\dagger$ This research was supported by the 2013 scientific promotion program funded by Jeju National University.

1 Assistant professor, Department of Computer Science and Statisitcs, Jeju National University, Jeju 690-756, Korea.

2 Corresponding author: Assistant professor, Department of Statistics and Actuarial Science, Soongsil University, Seoul 156-43, Korea. E-mail: bko@ssu.ac.kr 
series practice has often provided substantial evidence that these assumptions may be inadequate. For example, it is widely recognized that financial time series frequently exhibit time-varying volatility, skewness, and excess kurtosis which can not be captured by i.i.d innovations. Accordingly, since autoregressive conditional heteroscedastic (ARCH) model and generalized ARCH (GARCH) model were developed by Engle (1982) and Bollerslev (1986), ARMA models incorporating GARCH innovations are commonly preferred in empirical financial data analysis. For the reference on ARMA-GARCH models, we refer to Ling and Li (1998), Ling and McAleer (2003a, 2003b), and Francq and Zakoïan (2004). For the change points test in ARMA-GARCH models, refer to Lee and Song (2008), in which they developed the CUSUM test in ARMA-GARCH models by following the test procedure of Lee et al. (2003) together with the quasi-maximum likelihood estimator (QMLE) of Francq and Zakoïan (2004). Their test is reviewed in Section 2. For the change points test in GARCH models, we refer to Kim et al. (2000), Lee et al. (2004), and Berkes et al. (2004).

In this paper, we analyze the Korean Won/Japanese 100 Yen (KRW/JPY) exchange rate data based on ARMA-GARCH model and perform the CUSUM test of Lee and Song (2008) for detecting parameter changes. For practical prediction of exchange rate, we refer to Kim and Kwon (2011).

\section{CUSUM test for ARMA-GARCH models}

Let us consider the following $\operatorname{ARMA}(P, Q)$ models with $\operatorname{GARCH}(p, q)$ innovations:

$$
\begin{aligned}
& X_{t}-c_{0}=\sum_{i=1}^{P} a_{0 i}\left(X_{t-i}-c_{0}\right)+e_{t}-\sum_{j=1}^{Q} b_{0 j} e_{t-j}, \\
& \left\{\begin{array}{l}
e_{t}=\sqrt{h_{t}} \eta_{t}, \\
h_{t}=\omega_{0}+\sum_{i=1}^{p} \alpha_{0 i} e_{t-i}^{2}+\sum_{j=1}^{q} \beta_{0 j} h_{t-j},
\end{array}\right.
\end{aligned}
$$

where $\omega_{0}>0, \alpha_{0 i} \geq 0, \beta_{0 j} \geq 0$ and $\left\{\eta_{t}: t \in \mathbb{Z}\right\}$ is a sequence of independent and identically distributed random variables with zero mean and unit variance. We set $\varphi=$ $\left(\vartheta^{T}, \theta^{T}\right)^{T} \in \Phi \subset \mathbb{R}^{P+Q+1} \times(0, \infty) \times[0, \infty)^{p+q}$, where $\vartheta=\left(c, a_{1}, \cdots, a_{P}, b_{1}, \cdots, b_{Q}\right)^{T}, \theta=$ $\left(\omega, \alpha_{1}, \cdots, \alpha_{p}, \beta_{1}, \cdots, \beta_{q}\right)^{T}$, and denote the true parameter by $\varphi_{0}=\left(\vartheta_{0}^{T}, \theta_{0}^{T}\right)^{T}$.

In this paper, we intend to test the hypotheses

$$
\begin{aligned}
& H_{0}: \text { The true parameter } \varphi_{0} \text { does not change. vs. } \\
& H_{1} \text { : not } H_{0} \text {. }
\end{aligned}
$$

In order to perform a test, we employ the CUSUM test proposed by Lee and Song (2008):

$$
T_{n}=\max _{1 \leq k \leq n} \frac{k^{2}}{n}\left(\hat{\boldsymbol{\varphi}}_{k}-\hat{\boldsymbol{\varphi}}_{n}\right)^{T} \hat{\Sigma}_{n}\left(\hat{\boldsymbol{\varphi}}_{k}-\hat{\boldsymbol{\varphi}}_{n}\right):=\max _{1 \leq k \leq n} D_{k} .
$$

Here $\hat{\varphi}_{n}$ is the the quasi-maximum likelihood estimator (QMLE) of Francq and Zakoïan (2004) defined by

$$
\hat{\boldsymbol{\varphi}}_{n}=\arg \min _{\boldsymbol{\varphi} \in \Phi} \frac{1}{n} \sum_{t=1}^{n}\left(\frac{\tilde{\epsilon}_{t}^{2}(\vartheta)}{\tilde{\sigma}_{t}^{2}(\boldsymbol{\varphi})}+\log \tilde{\sigma}_{t}^{2}(\boldsymbol{\varphi})\right):=\arg \min _{\boldsymbol{\varphi} \in \Phi} \frac{1}{n} \sum_{t=1}^{n} \tilde{l}_{t}(\boldsymbol{\varphi}),
$$


and $\hat{\Sigma}_{n}$ is a consistent estimator of $\mathcal{J} \mathcal{I}^{-1} \mathcal{J}$, where

$$
\mathcal{J}=-\mathbb{E}\left\{\frac{\partial^{2} l_{t}\left(\boldsymbol{\varphi}_{0}\right)}{\partial \boldsymbol{\varphi} \partial \varphi^{T}}\right\} \quad \text { and } \quad \mathcal{I}=\mathbb{E}\left\{\frac{\partial l_{t}\left(\boldsymbol{\varphi}_{0}\right)}{\partial \boldsymbol{\varphi}} \frac{\partial l_{t}\left(\boldsymbol{\varphi}_{0}\right)}{\partial \boldsymbol{\varphi}^{T}}\right\}
$$

$\left\{\tilde{\epsilon}_{t}: 1 \leq t \leq n\right\}$ and $\left\{\tilde{\sigma}_{t}^{2}: 1 \leq t \leq n\right\}$ are the processes defined recursively by

$$
\begin{aligned}
& \tilde{\epsilon}_{t}:=\tilde{\epsilon}_{t}(\vartheta)=X_{t}-c-\sum_{i=1}^{P} a_{i}\left(X_{t-i}-c\right)+\sum_{j=1}^{Q} b_{j} \tilde{\epsilon}_{t-j}, \\
& \tilde{\sigma}_{t}^{2}:=\tilde{\sigma}_{t}^{2}(\boldsymbol{\varphi})=\omega+\sum_{i=1}^{p} \alpha_{i} \tilde{\epsilon}_{t-i}^{2}+\sum_{j=1}^{q} \beta_{j} \tilde{\sigma}_{t-j}^{2}
\end{aligned}
$$

and the initial values are assumed to be given properly. For simplicity, these initial values are fixed, neither random nor a function of the parameters. As estimators of $\mathcal{J}$ and $\mathcal{I}$, we consider using

$$
\hat{\mathcal{J}}_{n}=-\frac{1}{n} \sum_{t=1}^{n} \frac{\partial^{2} \tilde{l}_{t}\left(\hat{\boldsymbol{\varphi}}_{n}\right)}{\partial \boldsymbol{\varphi} \partial \boldsymbol{\varphi}^{T}} \quad \text { and } \quad \hat{\mathcal{I}}_{n}=\frac{1}{n} \sum_{t=1}^{n} \frac{\partial \tilde{l}_{t}\left(\hat{\boldsymbol{\varphi}}_{n}\right)}{\partial \boldsymbol{\varphi}} \frac{\partial \tilde{l}_{t}\left(\hat{\boldsymbol{\varphi}}_{n}\right)}{\partial \boldsymbol{\varphi}^{T}}
$$

Under $H_{0}$ and some regularity conditions, Lee and Song (2008) showed that the test statistic $T_{n}$ weakly converges to the sup of the square of a $(P+Q+p+q+2)$-dimensional Brownian bridge. That is,

$$
T_{n} \stackrel{w}{\longrightarrow} \sup _{0 \leq s \leq 1}\left\|W_{D}^{0}(s)\right\|_{2}^{2}
$$

where $D=P+Q+p+q+2$ and $W_{D}^{0}$ is a $D$-dimensional Brownian bridge. Thus, we reject $H_{0}$ if $T_{n}$ is large. The empirical $(1-\alpha)$ quantile values for $\sup _{0<s<1}\left\|W_{D}^{o}(s)\right\|_{2}^{2}, \alpha \in$ $\{0.01,0.05,0.1\}, D \in\{1, \ldots, 10\}$, can be found in Table 1 of Lee et al. (2003, pp.784).

For the purpose of comparison, the residual-based CUSUM test is also considered. That is, letting $\left\{\hat{e}_{t}\right\}$ be the sequence of the residuals of model (2.1) and (2.2), the test is conducted based on the following test statistics:

$$
T_{n}^{r e s}=\frac{1}{\sqrt{n} \hat{\tau}} \max _{1 \leq k \leq n}\left|\sum_{t=1}^{k} \hat{e}_{t}^{2}-\frac{k}{n} \sum_{t=1}^{n} \hat{e}_{t}^{2}\right|,
$$

where $\hat{\tau}^{2}=\frac{1}{n} \sum_{t=1}^{n} \hat{e}_{t}^{4}-\left(\frac{1}{n} \sum_{t=1}^{n} \hat{e}_{t}^{2}\right)^{2}$. To the authors' knowledge, the asymptotic distribution of the test statistics $T_{n}^{r e s}$ is not yet established in ARMA-GARCH models, but one can easily surmise that $T_{n}^{r e s}$ weakly converges to $\sup _{0 \leq s \leq 1}\left|W^{0}(s)\right|$, where $\left\{W^{0}(s)\right\}$ is a Brownian bridge, because $\hat{e}_{t}$ 's are expected to behave like i.i.d. random variables under the null hypothesis. Based on this conjecture, we undertake the residual-based test additionally. For the residual-based CUSUM test in GARCH models, we refer to Lee et al. (2004).

\section{Empirical data analysis}

In this section, we analyze a daily exchange rate series. The data employed in this application is KRW/JPY exchange rate data, which covers the period ranging from 4 January 2000 
to 28 December 2012, for a total of 3217 observations. The KRW/JPY exchange rate series and its $\log$ return series, that is $r_{t}=100 \log \left(s_{t} / s_{t-1}\right)$, are plotted in Figure 3.1. The elementary statistics and Jarque-Bera statistics (JB) are presented in Table 3.1, from which we can see that the return series shows a very high value in kurotis and thus the null hypothesis for testing the normality is rejected.
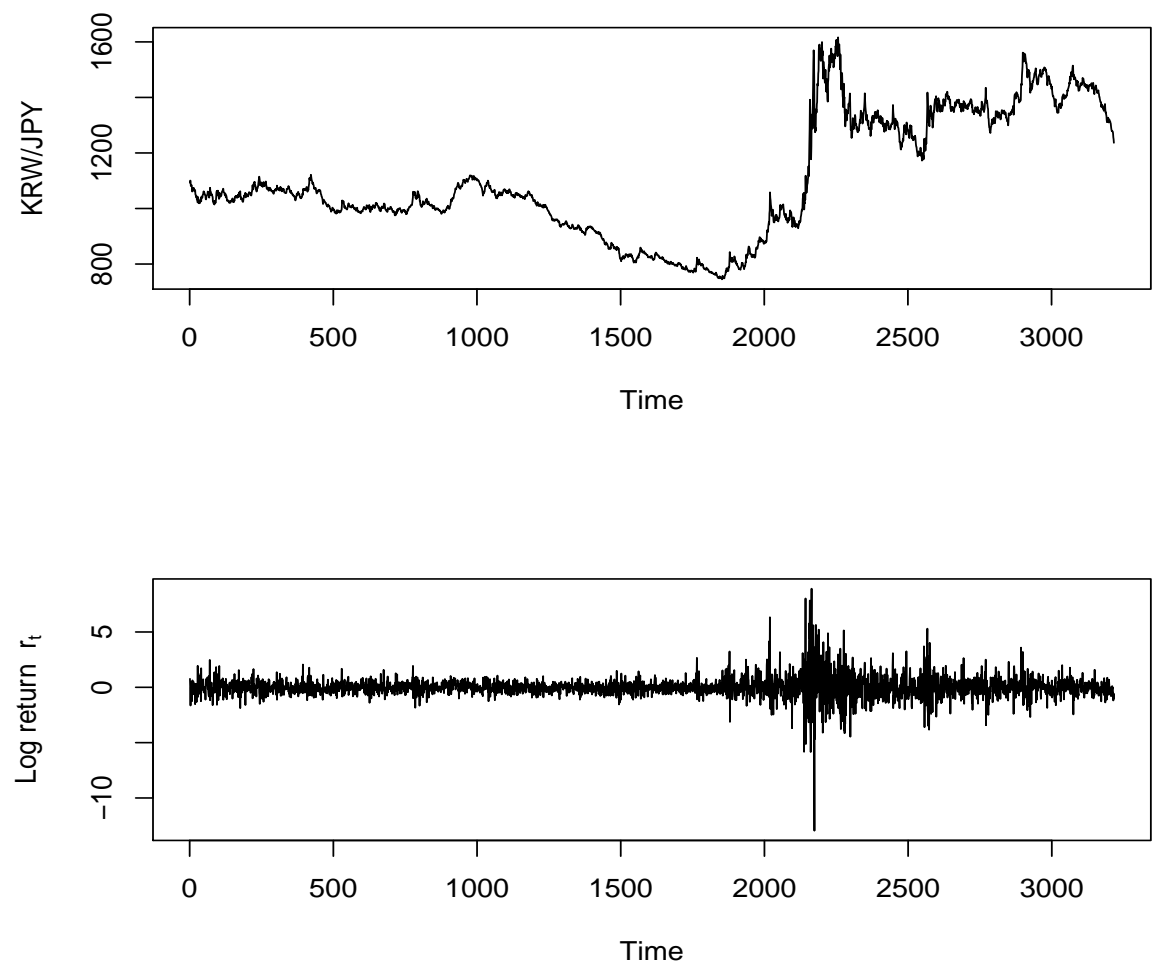

Figure 3.1 The KRW/JPY series and the log return series from Jan. 4, 2000 to Dec. 28, 2012

Table 3.1 Descriptive statistics for return series $\left\{r_{t}\right\}$

\begin{tabular}{cccccc}
\hline \hline Mean (s.d) & Min & Max & Skewness & Kurtosis & $J B$ \\
\hline $0.38 \%(0.96 \%)$ & $-12.96 \%$ & $8.91 \%$ & 0.18 & 21.56 & $62417[<0.000]$ \\
\hline
\end{tabular}

In order to check whether there occured some parameter changes in this period, we apply the above CUSUM test to the log return series $\left\{r_{t}\right\}$. First, we make a preliminary analysis of the whole return series. For selecting a model for the mean part of $\left\{r_{t}\right\}$, we rely on the Bayesian Information Criterion (BIC). The list of the ARMA models considered and the corresponding BIC values are presented in Table 3.2. Since AR(4) model produces the minimum $\mathrm{BIC}$ value, we take the $\mathrm{AR}(4)$ model for the mean part. 
Table 3.2 The ARMA models considered and the corresponding BIC values

\begin{tabular}{ccccccc}
\hline \hline & AR0 & AR1 & AR2 & AR3 & AR4 & AR5 \\
\hline MA0 & 8876.41 & 8884.14 & 8885.34 & 8880.07 & 8873.33 & 8879.32 \\
MA1 & 8884.11 & 8891.80 & 8889.44 & 8874.28 & 8879.49 & 8887.39 \\
MA2 & 8886.73 & 8890.64 & 8889.21 & 8880.04 & 8887.52 & 8892.36 \\
MA3 & 8879.48 & 8874.34 & 8882.32 & 8887.33 & 8895.48 & 8903.20 \\
MA4 & 8873.51 & 8880.76 & 8888.81 & 8895.57 & 8903.06 & 8911.12 \\
MA5 & 8880.69 & 8887.86 & 8895.93 & 8902.93 & 8910.98 & 8918.94 \\
\hline
\end{tabular}

For modeling the variance part of $\left\{r_{t}\right\}$, we compute the residuals $\left\{\hat{\epsilon}_{t}\right\}$ after estimating the parameters in AR(4) model through the least squares estimation (LSE). The ACFs for $\left\{\hat{\epsilon}_{t}\right\}$ and $\left\{\hat{\epsilon}_{t}^{2}\right\}$ presented in Figure 3.2 show that there is almost insignificant autocorrelation in $\left\{\hat{\epsilon}_{t}\right\}$ itself, but significantly positive autocorrelation in the squared residuals $\left\{\hat{\epsilon}_{t}^{2}\right\}$, which is a typical feature of ARCH-effect. Also, the results of the Ljung-Box test for the residuals and the squared residuals strongly indicate the existence of the ARCH-effect (See Table 3.3 below).
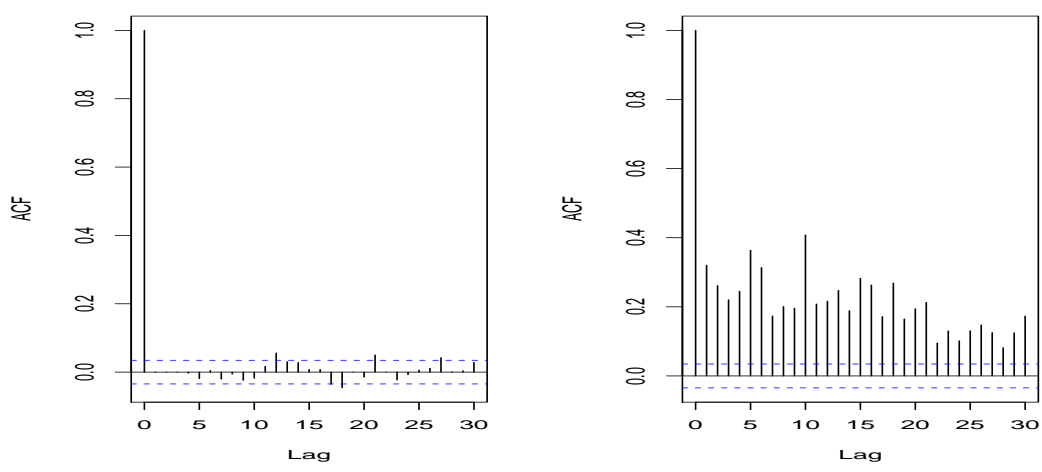

Figure 3.2 The ACF for the residuals $\left\{\hat{\epsilon}_{t}\right\}$ and the squared residuals $\left\{\hat{\epsilon}_{t}^{2}\right\}$

To deal with the ARCH-effect, we employ the $\operatorname{GARCH}(1,1)$ model as is usually done in most empirical studies for reasons of simplicity. In terms of the results of the LSE, which is given in Table 3.3, we choose a tentative model for the whole return series as $\operatorname{AR}(4)$ $\operatorname{GARCH}(1,1)$ model without the first AR parameter:

$$
r_{t}=\phi_{2} r_{t-2}+\phi_{3} r_{t-3}+\phi_{4} r_{t-4}+\epsilon_{t}, \quad\left\{\begin{array}{l}
\epsilon_{t}=\sqrt{h_{t}} \eta_{t}, \\
h_{t}=\omega+\alpha \epsilon_{t-1}^{2}+\beta h_{t-1}
\end{array},\right.
$$

where we assume that $\eta_{t}$ 's are i.i.d. random variables with zero mean and unit variance. The results of LSE and QMLE, and the Ljung-Box statistics for residuals and the squared residuals are reported in Table 3.3. As we can see, the sum of GARCH parameters, i.e., $\alpha+\beta$ is estimated to be close to one, which indicates that the fitted model appears to be an almost $\operatorname{IGARCH}(1,1)$. Here, it needs to be noted that the $\operatorname{GARCH}(1,1)$ model with parameter changes can be regarded as an $\operatorname{IGARCH}(1,1)$ model; see, for example, Lee et al. (2004) and Park and Lee (2007). 
Table 3.3 Estimation results and Ljung-Box statistics for $\left\{\hat{\epsilon}_{t}\right\}$ and $\left\{\hat{\epsilon}_{t}^{2}\right\}$

\begin{tabular}{cccc}
\hline \hline & LSE & QMLE & \\
\hline$\phi_{1}$ & $0.009(0.018)$ & $\phi_{1}$ & $-0.023(0.020)$ \\
$\phi_{2}$ & $0.045(0.018)^{*}$ & $\phi_{2}$ & $0.034(0.019)^{*}$ \\
$\phi_{3}$ & $-0.068(0.018)^{*}$ & $\phi_{3}$ & $0.023(0.019)$ \\
$\phi_{4}$ & $-0.066(0.018)^{*}$ & $\phi_{4}$ & $-0.028(0.019)$ \\
$Q_{\hat{\epsilon}}(10)$ & $5.590[0.848]$ & $\omega$ & $0.006(0.003)^{*}$ \\
$Q_{\hat{\epsilon}^{2}}(10) 2525.880[<0.000]$ & $\alpha$ & $0.101(0.021)^{*}$ \\
& & & $\beta$
\end{tabular}

Note: Standard errors are in parentheses. ${ }^{*}$ significant at the level $10 \%$.

Based on model (3.1), we conduct the CUSUM test in Section 2 at the nominal level 0.01. The test statistics $T_{n}$ is obtained to be 22.84 , which is larger than the critical value of 5.47 and hence the null hypothesis is rejected. Since the maximum value of $D_{k}$ is obtained at $k=1916$, we perform again the CUSUM test on the period before $t=1916$, based on newly specified model. Repeating the test procedure, we find the first change point at $t=107$, which corresponds to 13 June 2000. Then, we perform the test on the remaining period from 13 June 2000 to 28 December 2012. In each test, the model is newly specified by the BIC and the CUSUM test is conducted under the specified model with the insignificant coefficients omitted. For example, in the fourth period in Table 3.5, AR(4)-GARCH(1,1) model without the first two AR parameters is employed in the CUSUM test. Total nine CUSUM tests are conducted in our analysis until no change point is detected and we can find two more change points. The result of each CUSUM test is summarized in Table 3.4.

Table 3.4 The CUSUM test results

\begin{tabular}{lccccc}
\multicolumn{7}{c}{ Table 3.4 The CUSUM test results } \\
\hline \hline Period & Specified model & $T_{n}$ & Critical value & Result & Change point \\
\hline$[1: 3216]$ & ARMA(4,0)-GARCH(1,1) & 22.84 & 5.47 & reject $H_{0}$ & 1916 \\
{$[1: 1916]$} & ARMA(1,0)-GARCH(1,1) & 22.12 & 4.48 & reject $H_{0}$ & 107 \\
{$[1: 107]$} & ARMA(5,0) & 1.78 & 3.27 & accept $H_{0}$ & $\cdot$ \\
\hline$[107: 3216]$ & ARMA(4,0)-GARCH(1,1) & 92.18 & 5.47 & reject $H_{0}$ & 1786 \\
{$[107: 1786]$} & GARCH(1,1) & 5.91 & 3.90 & reject $H_{0}$ & 784 \\
{$[107: 784]$} & GARCH(1,1) & 1.06 & 3.90 & accept $H_{0}$ & $\cdot$ \\
\hline$[784: 3216]$ & ARMA(4,0)-GARCH(1,1) & 612.56 & 5.47 & reject $H_{0}$ & 1760 \\
{$[784: 1760]$} & GARCH(1,1) & 3.55 & 3.90 & accept $H_{0}$ & $\cdot$ \\
\hline$[1760: 3216]$ & ARMA(4,0)-GARCH(1,1) & 2.42 & 4.95 & accept $H_{0}$ & $\cdot$ \\
\hline
\end{tabular}

Table 3.5 Specified model and estimation result in each period

\begin{tabular}{|c|c|c|c|c|c|c|c|c|}
\hline Period & Specified Model & $\phi_{2}$ & $\phi_{3}$ & $\phi_{4}$ & $\phi_{5}$ & $\omega$ & $\alpha$ & $\beta$ \\
\hline $00 / 01 / 05 \sim 00 / 06 / 13$ & $\mathrm{AR}(5)$ & & & & $\begin{array}{c}0.190 \\
(0.089)\end{array}$ & $\begin{array}{c}0.579 \\
(0.097)\end{array}$ & & \\
\hline $00 / 06 / 13 \sim 03 / 03 / 19$ & $\operatorname{GARCH}(1,1)$ & & & & & $\begin{array}{c}0.058 \\
(0.021)\end{array}$ & $\begin{array}{c}0.096 \\
(0.042)\end{array}$ & $\begin{array}{c}0.684 \\
(0.097)\end{array}$ \\
\hline $03 / 03 / 19 \sim 07 / 02 / 23$ & $\operatorname{GARCH}(1,1)$ & & & & & $\begin{array}{c}0.109 \\
(0.042)\end{array}$ & $\begin{array}{c}0.145 \\
(0.045)\end{array}$ & $\begin{array}{c}0.300^{*} \\
(0.203)\end{array}$ \\
\hline $07 / 02 / 23 \sim 12 / 12 / 28$ & $\operatorname{AR}(4)-\operatorname{GARCH}(1,1)$ & & $\begin{array}{c}0.016^{*} \\
(0.028)\end{array}$ & $\begin{array}{c}-0.038^{*} \\
(0.028)\end{array}$ & & $\begin{array}{c}0.039 \\
(0.016)\end{array}$ & $\begin{array}{c}0.160 \\
(0.038)\end{array}$ & $\begin{array}{c}0.817 \\
(0.041)\end{array}$ \\
\hline $00 / 01 / 05 \sim 12 / 12 / 28$ & $\mathrm{AR}(4)-\mathrm{GARCH}(1,1)$ & $\begin{array}{c}0.032 \\
(0.019)\end{array}$ & $\begin{array}{c}0.021^{*} \\
(0.019)\end{array}$ & $\begin{array}{l}-0.027^{*} \\
(0.019)\end{array}$ & & $\begin{array}{c}0.006 \\
(0.003)\end{array}$ & $\begin{array}{c}0.102 \\
(0.021)\end{array}$ & $\begin{array}{c}0.892 \\
(0.023)\end{array}$ \\
\hline
\end{tabular}


Table 3.6 Estimation result of the full model AR(4)-GARCH $(1,1)$

\begin{tabular}{|c|c|c|c|c|c|c|c|}
\hline$\overline{\text { Period }}$ & $\overline{\phi_{1}}$ & $\overline{\phi_{2}}$ & $\overline{\phi_{3}}$ & $\overline{\phi_{4}}$ & $\bar{\omega}$ & $\alpha$ & $\bar{\beta}$ \\
\hline $00 / 01 / 05 \sim 00 / 06 / 13$ & -0.089 & 0.022 & -0.128 & -0.104 & $0.571^{*}$ & 0.000 & 0.015 \\
\hline & $(0.097)$ & $(0.083)$ & $(0.088)$ & $(0.103)$ & $(0.188)$ & $(0.000)$ & $(0.270)$ \\
\hline $00 / 06 / 13 \sim 03 / 03 / 19$ & $\begin{array}{c}-0.028 \\
(0.040)\end{array}$ & $\begin{array}{c}0.017 \\
(0.041)\end{array}$ & $\begin{array}{c}0.010 \\
(0.040)\end{array}$ & $\begin{array}{l}-0.018 \\
(0.040)\end{array}$ & $\begin{array}{c}0.060^{*} \\
(0.022)\end{array}$ & $\begin{array}{c}0.099^{*} \\
(0.045)\end{array}$ & $\begin{array}{c}0.674^{*} \\
(0.102)\end{array}$ \\
\hline $03 / 03 / 19 \sim 07 / 02 / 23$ & $\begin{array}{c}-0.034 \\
(0.037)\end{array}$ & $\begin{array}{c}0.044 \\
(0.034)\end{array}$ & $\begin{array}{c}0.061^{*} \\
(0.033)\end{array}$ & $\begin{array}{c}0.005 \\
(0.034)\end{array}$ & $\begin{array}{c}0.111^{*} \\
(0.047)\end{array}$ & $\begin{array}{c}0.130^{*} \\
(0.047)\end{array}$ & $\begin{array}{c}0.299 \\
(0.227)\end{array}$ \\
\hline $07 / 02 / 23 \sim 12 / 12 / 28$ & $\begin{array}{c}-0.008 \\
(0.029)\end{array}$ & $\begin{array}{c}0.030 \\
(0.030)\end{array}$ & $\begin{array}{c}0.017 \\
(0.028)\end{array}$ & $\begin{array}{c}-0.039 \\
(0.028)\end{array}$ & $\begin{array}{c}0.039^{*} \\
(0.016)\end{array}$ & $\begin{array}{c}0.161^{*} \\
(0.038)\end{array}$ & $\begin{array}{r}0.816^{*} \\
(0.041)\end{array}$ \\
\hline
\end{tabular}

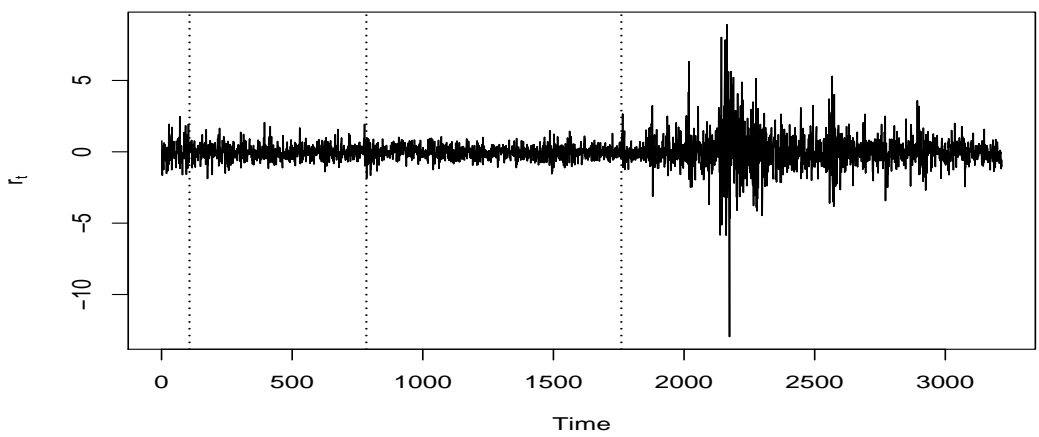

Figure 3.3 The log return series and change points in the period from Jan. 4, 2000 to Dec. 28, 2012

The change points and estimation results are presented in Table 3.5. While the whole series is finally fitted by $\operatorname{AR}(4)-\operatorname{IGARCH}(1,1)$ model with only the second AR parameter being significant, the return series in the first period is fitted by $\mathrm{AR}(5)$ model without the first four AR parameters and the rest actually by $\operatorname{GARCH}(1,1)$ model. In particular, we can see that the return series in the third period is fitted by $\mathrm{ARCH}(1)$ and the estimates of $\beta$ vary depending on the period: $\beta$ in the second, third, and fourth period are estimated to be $0.68,0.30$, and 0.82 , respectively. The estimates in Table 3.6, which reports the estimation result of $\mathrm{AR}(4)-\mathrm{GARCH}(1,1)$ in each period, appear similar to the values in Table 3.5, and thus we can conclude that the three change points divide the whole period meaningfully. Figure 3.3 presents the return series with the dashed vertical lines indicating the locations of the change points. It looks quite evident in Figure 3.3 that the return series before and after 23 February $2007(t=1760)$ have different appearances. As mentioned earlier, our empirical findings also show that if we ignore the change and fit the $\operatorname{GARCH}(1,1)$ model to the data in the whole period, the fitted model can be an almost $\operatorname{IGARCH}(1,1)$ model, which can lead to a false conclusion in statistical inference. Thus, inference such as forecasting should be not based on the results of the whole period, but based on the last period.

Table 3.7 The residual-based CUSUM test results

\begin{tabular}{lccccc}
\multicolumn{7}{c}{ Table 3.7 The residual-based CUSUM test results } \\
\hline \hline \multicolumn{1}{c}{ Period } & Specified model & $T_{n}^{\text {res }}$ & Critical value & Result & Change point \\
\hline$[1: 3216]$ & ARMA(4,0)-GARCH $(1,1)$ & 1.92 & 1.49 & reject $H_{0}$ & 1853 \\
{$[1: 1853]$} & GARCH $(1,1)$ & 1.56 & 1.49 & reject $H_{0}$ & 802 \\
{$[1: 802]$} & GARCH $(1,1)$ & 1.15 & 1.49 & accept $H_{0}$ & $\cdot$ \\
\hline$[802: 3216]$ & ARMA $(4,0)-G A R C H(1,1)$ & 1.74 & 1.49 & reject $H_{0}$ & 1829 \\
{$[802: 1829]$} & ARMA $(1,0)-G A R C H(1,1)$ & 0.87 & 1.49 & accept $H_{0}$ & $\cdot$ \\
\hline$[1829: 3216]$ & ARMA $(4,0)-G A R C H(1,1)$ & 1.40 & 1.49 & accept $H_{0}$ & $\cdot$ \\
\hline
\end{tabular}


Table 3.8 Specified model and estimation result in each period

\begin{tabular}{|c|c|c|c|c|c|c|c|c|}
\hline Period & Specified Model & $\overline{\phi_{1}}$ & $\overline{\phi_{2}}$ & $\phi_{3}$ & $\phi_{4}$ & $\omega$ & $\alpha$ & $\bar{\beta}$ \\
\hline $00 / 01 / 05 \sim 00 / 04 / 14$ & GARCH $(1,1)$ & & & & & $\begin{array}{c}0.019 \\
(0.009)\end{array}$ & $\begin{array}{c}0.072 \\
(0.026)\end{array}$ & $\begin{array}{c}0.870 \\
(0.048)\end{array}$ \\
\hline $03 / 04 / 14 \sim 07 / 06 / 05$ & $\operatorname{AR}(1)-\operatorname{GARCH}(1,1)$ & $\begin{array}{c}-0.046^{*} \\
(0.033)\end{array}$ & & & & $\begin{array}{c}0.025 \\
(0.013)\end{array}$ & $\begin{array}{c}0.103 \\
(0.032)\end{array}$ & $\begin{array}{c}0.768 \\
(0.093)\end{array}$ \\
\hline $07 / 06 / 05 \sim 12 / 12 / 28$ & $\operatorname{AR}(4)-\operatorname{GARCH}(1,1)$ & $\begin{array}{c}-0.006^{*} \\
(0.029)\end{array}$ & $\begin{array}{c}0.026^{*} \\
(0.030)\end{array}$ & $\begin{array}{c}0.011^{*} \\
(0.029)\end{array}$ & $\begin{array}{l}-0.036^{*} \\
(0.029)\end{array}$ & $\begin{array}{c}0.054 \\
(0.013)\end{array}$ & $\begin{array}{c}0.168 \\
(0.026)\end{array}$ & $\begin{array}{c}0.797 \\
(0.029)\end{array}$ \\
\hline
\end{tabular}

For the purpose of comparison, we also consider the residual-based CUSUM test. We note that the critical values are taken from the distribution of $\sup _{0 \leq s \leq 1}\left|W^{0}(s)\right|$ although the asymptotic distribution of $T_{n}^{r e s}$ in ARMA-GARCH model is not yet verified. The test is conducted at nominal level 0.1 because the residual-based tests have a tendency to show comparatively lower power than the estimates-based tests. As shown in Table 3.7, based on the residual-based test, two change points are detected: the first is 14 April 2003 and the second is 5 June 2007, which might correspond to the second and third change points in the results of Table 3.4. It seems that the first change point in Table 3.4 might not be detected in the residual-based test. Although AR-GARCH model is specified in the second and the third periods, all the AR parameters are estimated to be insignificant, and thus the whole return series are fitted by $\operatorname{GARCH}(1,1)$ model. Accordingly, we perform again the residual-based test based on the $\operatorname{GARCH}(1,1)$ model and it is found that its change points coincide with those in Table 3.7. Note that the $T_{n}^{r e s}$ in $\operatorname{GARCH}(1,1)$ model has the asymptotic distribution of $\sup _{0 \leq s \leq 1}\left|W^{0}(s)\right|$ under the null hypothesis; see Lee et al. (2004).

\section{Conclusion}

In this paper, we analyze the Korean Won/Japanese 100 Yen exchange rate data during the past decade under the ARMA-GARCH model. By employing the CUSUM test proposed in Lee and Song (2008), we could locate several parameter change points and partition the whole series into the ones which allow for stationary ARMA-GARCH modeling. As addressed in the previous literature, our empirical findings indicate that ARMA-GARCH $(1,1)$ models with parameter or structural changes can result in ARMA-IGARCH(1,1) models, in other words, parameter changes or structural breaks can be a reason of IGARCH modeling.

\section{References}

Berkes, I., Horváth, L., and Kokoszka, P. (2004). Testing for parameter constancy in GARCH(p,q) models. Statistics and Probability Letter, 4, 263-273.

Bollerslev, T. (1986). Generalized autoregressive conditional heteroskedasticity. Journal of Econometrics, 31, 307-327.

Chan, J. and Gupta, A. K. (2000). Parametric statistical change point analysis, Brokäuser, Boston.

Csörgö, M. and Horváth, L. (1997). Limit theorems in change-point analysis, John Wiley \& Sons Ltd, West Sussex, England.

Engle, R. F. (1982). Autoregressive conditional heteroskedasticity with estimates of the variance of United Kingdom inflation. Econometrica, 50, 987-1008.

Francq, C. and Zakoïan, J.-M. (2004). Maximum likelihood estimation of pure GARCH and ARMA-GARCH processes. Bernoulli, 10, 605-637.

Hamilton, J.D. (1994) Time series analysis, Princeton University Press, New Jersey.

Kim, S., Cho, S. and Lee, S. (2000). On the cusum test for parameter changes in GARCH(1,1) models. The Communications in Statistics, Theory and Methods, 29, 445-462. 
Kim, T. Y. and Kwon, O. (2011). Confidence interval forecast of exchange rate based on bootstrap method during economic crisis. Journal of the Korean Data $\&$ Information Science Society, 22, 895-902.

Kokoszka, P. and Leipus, R. (2000). Change-point estimation in ARCH models. Bernoulli, 6, 513-539.

Lee, S., Ha, J., Na, O. and Na, S. (2003). The cusum test for parameter change in time series models. Scandinavian Journal of Statistics, 30, 781-796.

Lee, S. and Song, J. (2008). Test for parameter change in ARMA models with GARCH innovations. Statistics and Probability Letter, $\mathbf{7 8}, 1990-1998$.

Lee, S., Tokutsu, Y. and Maekawa, K. (2004). The cusum test for parameter change in regression models with ARCH errors. Journal of Japan Statistical Society, 34, 173-188.

Ling, S. and Li, W. K. (1998). Limiting distributions of maximum likelihood estimators for unstable autoregressive moving-average time series with general autoregressive heteroscedastic errors. Annals of Statistics, 26, 84-125.

Ling, S. and McAleer, M. (2003a). Asymptotic theory for a vector ARMA-GARCH model. Econometric Theory, 19, 280-310.

Ling, S. and McAleer, M. (2003b). Adaptive estimation in nonstationry ARMA models with GARCH noises. Annals of Statistics, 31, 642-674.

Park, S., and Lee, S. (2007). Modelling KOSPI200 data based on $\operatorname{GARCH}(1,1)$ parameter change test. Journal of the Korean Data \& Information Science Society, 18, 11-16.

Picard, D. (1985). Testing and estimating change-points in time series. Advances in Applied Probability, 17, 841-867.

Winchern, D. W., Miller, R. B. and Hsu, D. A. (1976). Changes of variance in first-order autoregressive time series models with an application. Journal of the Royal Statistical Society C, 25, 248-256. 